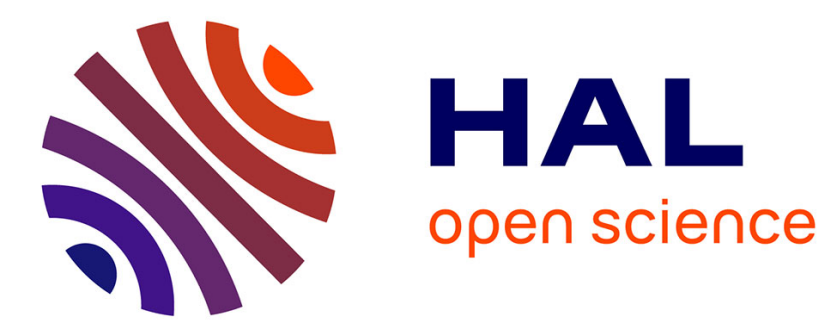

\title{
In vivo analysis of conjunctiva in gold micro shunt implantation for glaucoma
}

Leonardo Mastropasqua, Luca Agnifili, Marco Ciancaglini, Mario Nubile, Paolo Carpineto, Vincenzo Fasanella, Michele Figus, Stefano Lazzeri, Marco Nardi

\section{To cite this version:}

Leonardo Mastropasqua, Luca Agnifili, Marco Ciancaglini, Mario Nubile, Paolo Carpineto, et al.. In vivo analysis of conjunctiva in gold micro shunt implantation for glaucoma. British Journal of Ophthalmology, 2010, 94 (12), pp.1592. 10.1136/bjo.2010.179994 . hal-00572628

\section{HAL Id: hal-00572628 https://hal.science/hal-00572628}

Submitted on 2 Mar 2011

HAL is a multi-disciplinary open access archive for the deposit and dissemination of scientific research documents, whether they are published or not. The documents may come from teaching and research institutions in France or abroad, or from public or private research centers.
L'archive ouverte pluridisciplinaire HAL, est destinée au dépôt et à la diffusion de documents scientifiques de niveau recherche, publiés ou non, émanant des établissements d'enseignement et de recherche français ou étrangers, des laboratoires publics ou privés. 


\section{In vivo analysis of conjunctiva in gold micro shunt implantation for glaucoma}

Leonardo Mastropasqua,* Luca Agnifili,* Marco Ciancaglini, ** Mario Nubile,* Paolo Carpineto, * Vincenzo Fasanella, ${ }^{*}$ Michele Figus, $* * *$ Stefano Lazzeri, $* * *$ and Marco Nardi ***

*Department of Medicine and Ageing Science, Ophthalmic Clinic. University of Chieti-Pescara, Italy

**Department of Surgical Science, Ophthalmic Clinic. University of L'Aquila, Italy

***Department of Neuroscience, Ophthalmology. University of Pisa, Italy

Corresponding Author:

Luca Agnifili

Address: Via dei Frentani, 114

66100 Chieti, Italy

Fax: +39-0871-358794

Telephone: +39-0871-358410

E-mail: 1.agnifili@unich.it

Licence for Publication

The Corresponding Author has the right to grant on behalf of all authors and does grant on behalf of all authors, an exclusive licence (or non exclusive for government employees) on a worldwide basis to the BMJ Publishing Group Ltd to permit this article (if accepted) to be published in BJO and any other BMJPGL products and sublicences such use and exploit all subsidiary rights, as set out in our licence (http://group.bmj.com/products/journals/instructions-for-authors/licence-forms).

Competing Interest: None declared.

Key Words: conjunctiva, trans-scleral outflow, suprachoroidal space, gold micro shunt, in vivo confocal microscopy, primary open angle glaucoma.

Word count: 2895 


\begin{abstract}
Aims: To describe the conjunctival epithelial features seen with in vivo confocal microscopy (IVCM) after gold micro shunt (GMS) implantation in the suprachoroidal space, in patients with uncontrolled glaucoma.
\end{abstract}

Methods: This was an observational case series study. Fourteen eyes of 14 consecutive glaucomatous patients with a history of multiple failed incisional surgeries followed by GMS implantation, were evaluated with digital confocal Laser-Scanning Microscope (HRT II Rostock Cornea Module). Patients were divided into two groups: successful implantations (Group 1: eight patients, eight eyes), defined as a one-third reduction in preoperative intraocular pressure (IOP) with or without antiglaucoma medications and failed implantations (Group 2: six patients, six eyes) as a less than onethird reduction in preoperative IOP with maximal tolerated medical therapy. The examination was

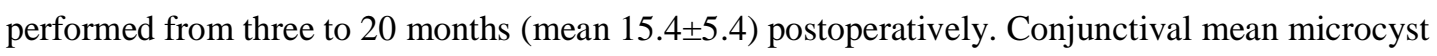
density (MMD: cysts $/ \mathrm{mm}^{2}$ ) and mean microcyst area (MMA: $\mu \mathrm{m}^{2}$ ) were the main outcome measurements.

Results: The mean post-operative IOP was statistically different between the 2 groups $(\mathrm{P}<0.05)$, with the values of $14.3 \pm 2.77$ and $32.3 \pm 8.01 \mathrm{mmHg}$ in Group 1 and 2, respectively.

When comparing successful with failed implantation, the IVCM analysis showed a greater MMD $(\mathrm{P}<0.01)$ and MMA $(\mathrm{P}<0.01)$. Clinical evidence of filtering bleb was not found in any of the patients. Conclusions: Successful GMS implantation significantly increased conjunctival microcysts density and surface at the site of the device insertion. These findings suggest that the enhancement of the aqueous filtration across the sclera may be one of the possible outflow pathway exploited by the shunt. 


\section{Introduction}

To date, filtration surgery and drainage device implantation are the most reliable procedures for lowering the intraocular pressure (IOP) in patients with medically uncontrolled glaucoma. ${ }^{1-4}$ The basic mechanism of these procedures is the creation of a new drainage route which allows aqueous humour to drain from the anterior chamber, thereby circumventing the pathological outflow obstruction. The aqueous then flows directly or indirectly into sub-conjunctival spaces leading to an elevation of the conjunctiva at the surgical site, which is commonly referred to as a filtering bleb. ${ }^{5,6}$ In a variable percentage of patients, either filtration procedures or drainage device implantation can lead to significant post-operative complications such as bleb leaks, hypotony, blebitis, endophthalmitis and bleb encapsulation, the latter being widely recognized as the main cause of surgical failure. ${ }^{7,8}$ Intense clinical and instrumental analysis of the bleb with frequent post-operative management still represents the main challenge in the maintenance of long term surgical success of all available filtration surgical procedures.

In the last years, bleb-less surgical approaches which exploit and enhance alternative aqueous outflow pathways have been evaluated in order to avoid these complications.

One such procedure involves the use of gold micro shunt (GMS) devices which are 24-karat gold, biocompatible flat plates containing micro-tubular channels that create a bridge between the anterior chamber (AC) and suprachoroidal space. Thus forcing aqueous flow through the two compartments, taking advantage of the natural gradient of hydrostatic pressure (from 1 to $5 \mathrm{mmHg}$ ) between the AC and suprachoroidal space, respectively. ${ }^{9}$ Hence, aqueous humour is removed via the choroidal vascular system or by gradually flowing through the scleral layers and then the conjunctiva, without the creation of a bleb. Recently, Melamed et al ${ }^{10}$ evaluated the efficacy and safety of GMS implantation in the supraciliay space in failed incisional glaucoma surgery, reporting a 79\% surgical success, without relevant post-operative complications and without the formation of filtering bleb in any of the patients. However, to best of our knowledge, no reports have specifically focused on the possible aqueous outflow pathways exploited by the GMS device.

The aim of the present study was to describe the bulbar conjunctival features in glaucomatous patients who underwent GMS implantation by using in vivo confocal microscopy (IVCM), in order to 
elucidate modifications induced by this device.

\section{Material and Methods}

This was an observational case series study. The study adhered to the tenets of the Declaration of Helsinki and informed consent was obtained from all patients prior to their enrollment. Our local Ethics Boards were notified which determined that their approval was not necessary. We examined 14 eyes ( 8 right and 6 left eyes) of 14 consecutive Caucasian patients affected with primary open angle glaucoma (POAG) who had been referred to the Ophthalmic Clinics of the Universities of ChietiPescara and Pisa, Italy (8 males and 6 females, with an age range from 43 to 77 years, mean 64.6 \pm 11.8 ). All patients had a history of multiple failed incisional procedures, followed by GMS implantation (SOLX, Boston, MA) from 10 to14 months after the last failed surgery.

The number of failed procedures prior to GMS implantation ranged from 1 to 2 (mean 1.4 \pm 0.5 ) and corresponded to MM-C augmented trabeculectomy (14 procedures) or deep sclerectomy with T-Flux ${ }^{\circledR}$ implant (6 procedures).

The GMS was inserted in the superior-temporal ( 9 eyes) or superior-nasal (5 eyes) region, as indicated on a patient-to-patient basis in order to avoid inadvertent intra-operative manipulations of the sites used in previous procedures. To comply with this intention, the conjunctiva overlying the site of the previous failed filtration procedure close to the planned site for GMS insertion, was pre-operatively marked (methylene blue) 2-3 mm outwardly to the limits of the presumed scleral flap. Therefore, the conjunctival flap and the scleral dissection for the GMS implantation were created at least $2 \mathrm{~mm}$ from delimited area.

A detailed description of the shunt and surgical technique have been previously reported. ${ }^{10}$ The time range of post-operative examinations was 3 to 20 months (mean: 15.4 \pm 5.4 mo) after GMS implantation.

Each patient, after a complete ophthalmologic assessment including visual acuity, applanation tonometry, anterior segment slit-lamp evaluation with particular attention at the conjunctiva overlying the site of implantation (a set of reference photographs was obtained in all cases) and fundoscopy, was carefully examined with a digital confocal Laser-Scanning Microscope (LSM) (HRT II Rostock 
Cornea Module, Heidelberg Engineering GmbH, Germany). Additionally, in order to verify the correct position of the proximal and distal end of the GMS implant, each patient underwent gonioscopy and anterior segment optical coherence tomography (AS-OCT) (Carl Zeiss Meditec, Inc, Dublin, CA). According to the achievement of satisfactory post-operative IOP control and on evidence of surgery success, ${ }^{11}$ GMS implantations were classified in 2 groups: successful implantations (qualified and complete success) (Group 1; defined as a third reduction in preoperative IOP with or without antiglaucoma treatment ${ }^{12}$ ) and failed implantations (Group 2; as a reduction lower than a third in preoperative IOP in maximal tolerated medical therapy).

All the patients had been on preserved topical antiglaucoma medications; the mean duration of topical therapy and the drug regimen before GMS implantation in each group, were reported in Table 1.

During the three months before IVCM examination, none of the patients received systemic or topical therapy (steroids) which could modify ocular hydrodynamics .

\section{In vivo confocal microscopy}

The technical characteristics of the instrument and the details of conjunctival examination were previously described. ${ }^{13}$ In this case series we investigated the microscopic epithelial features of the bulbar conjunctiva overlying the location of the GMS implantation.

In order to avoid in the field of analysis the site of the previous surgery close to the device location, we marked (methylene blue) the margins of the conjunctiva overlying the site of the GMS implantation (delineating a $4 \times 5 \mathrm{~mm}$ area $\left(20 \mathrm{~mm}^{2}\right)$, at least $2-3 \mathrm{~mm}$ from site of the previous surgery) and performed the IVCM at the centre of the selected area $\left(2 \times 3 \mathrm{~mm}\left(6 \mathrm{~mm}^{2}\right)\right)$.

IVCM analysis of each eye was performed by a single operator (VF) who selected six images (from about 40 images) which were evaluated and the results averaged by a second IVCM operator (LA). Both operators were masked for patient surgical history and status.

The patient was seated in front of the microscope, head steadied by a headrest and with the eye properly aligned using a dedicated target mobile bright red light provided with the instrument that the patient had to fix with the fellow eye, in order to obtain a tangential optical section of the superior bulbar conjunctiva. A digital camera was used to obtain am image of the lateral view of the eye and objective lens in order to check the position of the latter on the surface of the eye for each scan. LSM 
objective was gently positioned in contact with the ocular surface separated by a PMMA (polymethyl methacrylate) contact cap and a drop of $0.2 \%$ polyacrylic gel (Viscotirs® Gel, CIBA Vision ${ }^{\circledR}$ Ophthalmics, Marcon, Venezia, Italy) served as coupling medium. Sequential images 300X300 $\mu \mathrm{m}$ in size derived from automatic scans and manual frames were acquired at the intermediate layer of the bulbar conjunctival epithelium (10 to $20 \mu \mathrm{m}$ ), $2 \mathrm{~mm}$ from the limbus at the centre of the corresponding site of the GMS implantation, with the eye in opposite gaze with respect to site of the implant.

For the IVCM assessment, we evaluated the microcysts in the bulbar conjunctival epithelium, as first reported in the conjunctival wall of functioning filtering blebs. ${ }^{14-17}$ The main parameters considered were the mean microcyst density (MMD) $\left(\right.$ cysts $\left./ \mathrm{mm}^{2}\right)$ and the mean microcyst area (MMA) $\left(\mu \mathrm{m}^{2}\right)$. The total surface area of epithelial microcysts was calculated in each of the 6 selected confocal images by means of ImageJ, an open source software (http://rsb.info.nih.gov/ij), using previous described methods. $^{15}$

\section{Statistical analysis}

Statistical analysis was performed using SPSS $^{\circledR}$ Advanced Statistical $^{\mathrm{TM}}$ 13.0 Software (2005, Chicago, IL, USA). Differences in age and gender in successful and failed groups at baseline were evaluated using Student's t test and chi-squared, respectively. Unpaired $t$ test was used to analyze pre- and postoperative IOP, pre- and post-operative number of medications, duration of preoperative therapy and time of IVCM assessment after surgery between the two groups.

Paired t test was used to analyze the changes of post-operative of IOP or the number of medications in each group. U Mann-Whitney test was used to assess differences on conjunctival microcysts density and surface between the two groups.

For the given effect size the power of the study was 0.81 and 0.82 for microcysts density and area, respectively (Sample Power. SPSS ${ }^{\circledR}$ Advanced Statistical $^{\mathrm{TM}}$ 13.0 Software (2005, Chicago, IL, USA)).

\section{Results}

The demographic and clinical data of each group are shown in Table 1. No significant differences 
were found in age, sex, preoperative IOP, follow-up period and the time range of post-operative examinations (months) between the 2 groups. All reported data corresponded to the last follow-up visit (15.4 \pm 5.4 months), which matched with the IVCM examination.

The mean overall (Group 1 and 2) post-operative IOP percentage reduction was $22.6 \%$, from $28.8 \pm 3.9$ $\mathrm{mmHg}$ to $22.1 \pm 10.6 \mathrm{mmHg}(\mathrm{p}<0.05)$.

Mean post-operative IOP was statistically different between the two groups $(\mathrm{p}=0.002)$, with values of $14.3 \pm 2.8$ and $32.3 \pm 8.0 \mathrm{mmHg}$ in Groups 1 and 2, respectively.

In Group 1, mean IOP decreased from $28.1 \pm 5.0$ to $14.3 \pm 2.8 \mathrm{mmHg}$ (percentage reduction of $50.6 \%$ ) $(\mathrm{p}<0.001)$, while we did not observe statistically significant modifications in Group 2.

The drug dose regimen and the duration of preoperative therapy was not statistically different between groups. The mean number of antiglaucoma medications at baseline and at the last follow-up was not statistically different between groups; these values were not significantly modified in the post-operative period in either group: from $2.7 \pm 0.9$ to $2.2 \pm 1.0$ in Group 1 and $3.0 \pm 0.6$ to $2.8 \pm 0.4$ in Group 2 . In both groups the clinical assessment (according to MBGS criteria), ${ }^{18}$ the evaluation of the set of the reference photographs and the AS-OCT images did not show evidence of filtering bleb at the current surgical site in any case (Figures 1 and 2, A and B). In all eyes, as confirmed by gonioscopy and ASOCT examination, the device was correctly positioned in both the AC and the suprachoroidal space with an appearance of spongy or tight overlying sclera (Figures 1 and 2, B). Additionally, AS-OCT showed an hypoechoic space posteriorly to the shunt in several successful implantations (Fig. 1,B)

When analyzing the considered IVCM parameters, we found a statistically significant difference between the 2 groups, either for the microcysts density $\left(\right.$ cysts $\left./ \mathrm{mm}^{2}\right)$ or area $\left(\mu \mathrm{m}^{2}\right)($ Table 2$)(\mathrm{p}<0.01)$. Particularly, we did find values of $103.1 \pm 22.6$ and $29489.3 \pm 12954.9$ and $22.4 \pm 11.9$ and 4696.0 \pm 3608.1 for MMD and MMA in functioning and failed implantations, respectively (Figures 1 and 2, C).

\section{Discussion}

The scarring of conjunctival filtering bleb is the main challenge and limiting factor for long-term IOP control in glaucoma surgery. The bulk of the scientific evidences, strongly support that all anti- 
glaucoma procedures which create a fistula and route aqueous humour from AC to sub-conjunctival spaces, are exposed to a significant risk of bleb scarring and failure over time. ${ }^{8,19}$ The pathophysiological basis of the bleb scarring appear to be related to the action of a variety of factors present in the aqueous humour of glaucomatous eyes, which are capable of stimulating a fibrotic response after contact with the vascular Tenon's tissue. ${ }^{20}$ Additionally, fibrosis may be mechanically activated by the immediate post-surgical aqueous filtration into the sub-conjunctival space, as demonstrated after the implantation of Ahmed devices. ${ }^{21}$

GMS (now commercially available only as Plus version) is one of the developing surgical procedures which works by shunting fluids from AC to suprachoroidal space, thus avoiding the direct and immediate post-operative aqueous outflow into sub-conjunctiva and the creation of a bleb. In the present study we analyzed the microscopic features of the bulbar conjunctiva at surgical site with particular attention to intra-epithelial microcysts, in patients who underwent uncomplicated GMS implantation after the failure of previous filtration surgeries.

Concerning the post-operative IOP, an average global IOP reduction of $22.6 \%$ from baseline was found at the last follow-up (15.4 \pm 5.42 months) and all successful patients were classified in qualified success.

These results are quite different with respect to those reported by Melamed et al. ${ }^{10}$ who found a postoperative IOP decrease and complete success of $32.6 \%$ and $13.2 \%$, respectively. Such inconsistency could result from the surgical history of the patients: Melamed et al. included in their series either patients who had previous glaucoma surgery, a glaucoma drainage device $(53 \%)$ or patients who did not, while in our series all patients underwent at least one previous filtration surgery and were to be considered refractory glaucoma.

Epithelial microcysts are empty optically clear spaces originally described in the conjunctival bleb wall of successful trabeculectomies, as hallmarks of humor aqueous filtration through the conjunctiva. ${ }^{14}$ Recently Amar and coll., ${ }^{22}$ using both impression cytology and IVCM, modified and improved the definition of the bleb wall microcysts demonstrating that such structures correspond to goblet cells, mostly containing aqueous humor instead of highly hydrophilic gel-forming mucin. Based on these findings, the authors suggested that the trans-cellular pathway of the aqueous humor could 
occur at the level of goblet cells toward the ocular surface. More interestingly, in others studies ${ }^{23,24}$ the presence of intra-epithelial microcysts was documented also in eyes affected with untreated ocular hypertension or medically treated POAG that had not undergone surgical procedures. These authors have speculated that these findings support the hypothesis of an activation and/or enhancement of alternative hydrodynamic pathways and, specifically, the trans-scleral outflow, in conditions of increased IOP.

In the present study, when comparing functioning with non functioning implantations we did find a statistically significant difference for both mean microcysts density and surface, with values approximately five fold and six fold higher.

At the biomicroscopic examination none of the implanted patients developed a post-operative filtering bleb, indicating the absence of a direct post-operative sub-conjunctival aqueous filtration just as the technique of implantation require. Therefore, the evidence of conjunctival microcysts at the site of successful GMS implantation might be interpreted as a sign of aqueous percolation through the scleral layers and then the conjunctiva.

Based on the results of a previous report ${ }^{23}$ which ruled out an active role of topical anti-glaucoma medications in the conjunctival microcysts formation, the effects of topical drugs on IVCM considered parameters, was not investigated. Moreover, signs of inflammatory cells were not reported as significant features in the majority of cases, although this aspect was not methodically investigated. To date, the main mechanisms involved in the IOP reduction after GMS implantation are yet to be determined. However, our results may validate the assumption of Melamed et $\mathrm{al}^{10}$ who supposed that, once aqueous humor reaches the suprachoroidal space, it drains into the choroidal vascular system or permeates through the sclera. In support of this, the hypoechoic space sometimes observed posteriorly to the functioning implants, may indicate the suprachoroidal outflow pathway. Conversely, both the evidence of epithelial microcysts and the spongy appearance of the sclera at IVCM and AS-OCT examinations may be interpreted as the microscopic and macroscopic signs of the supposed activation of the trans-scleral aqueous outflow in functioning GMS shunts.

The patho-physiological basis of this mechanism is most likely an effect of the GMS introduction into the AC. This action technically require a scleral dissection and, therefore, the creation of a plane of 80- 
$90 \%$ scleral thickness. The final result is a partial scleral thinning with a reduction of the trans-scleral fluid movement resistivity which permits aqueous humor to move easier and in larger amount from suprachoroidal space to sub-conjunctiva.

In support of this, the importance and the role of the scleral thinning in filtration surgery success, has been recently demonstrated also in eyes that underwent MM-C augmented trabeculectomy. ${ }^{24}$ By considering such results, bleb-less surgical approaches which shunt aqueous humour to suprachoroidal space and/or thin the sclera thus increasing the trans-scleral outflow, could be useful to improve the success of antiglaucoma surgical procedures and avoid the intensive post-operative management.

However, as Melamed et al, we did not calculate the exact contribution of the suprachoroidal aqueous resorption and trans-scleral outflow to the mechanism of IOP reduction.

In non functioning cases, various mechanisms of failure may be hypothesized. The most relevant could be the formation of a fibrotic capsule around the device isolating the shunt from the choroidal vascular bed and the sclera. Alternatively, a connective invasion and/or an occlusion of the proximal end channels and the distal end holes or both mechanisms should be evoked. The effect is a proximal or a distal blockage of the aqueous outflow, therefore reaching and permeating the sclera would be unfeasible. These aspects could justify the very low density of epithelial microcysts at IVMC and the tight feature of the sclera at AS-OCT in failed implantations. Nevertheless, to verify our hypothesis, histological studies aimed at the analysis of the removed shunts in non functioning cases are mandatory.

The main limitation of our study is the lack of information regarding the status of conjunctival epithelium before GMS surgery, which may be useful to accurately calculate the trans-scleral aqueous flow enhancement achieved with the shunt, as recently verified for trabeculectomy. ${ }^{24}$ Furthermore, to avoid reading biases due to the effect of the previous filtration procedures on the overall trans-scleral outflow, the effects of GMS should be evaluated in glaucomatous patients naïve for glaucoma surgery. In conclusion, the in vivo analysis of conjunctiva in glaucomatous patients implanted with GMS provided valuable information which may contribute to clarify patho-physiological aspects of the aqueous outflow pathways exploited by the shunt. 


\section{References}

1. Jones E, Clarke J, Khaw PT. Recent advances in trabeculectomy technique. Curr Opin Ophthalmol 2005;16:107-13.

2. Hodkin MJ, Goldblatt WS, Burgoyne CF, et al. Early clinical experience with the Baerveldt implant in complicated glaucomas. Am J Ophthalmol 1995;120:32-40.

3. Mermoud A, Salmon JF, Alexander P, et al. Molteno tube implantation for neovascular glaucoma. Long-term results and factors influencing the outcome. Ophthalmology 1993;100:897902.

4. Coleman AL, Hill R, Wilson MR, et al. Initial clinical experience with the Ahmed Glaucoma Valve implant. Am J Ophthalmol 1995;120:23-31.

5. Benedikt O. The effects of filtering operations. Klin Monatsbl Augenheilkd 1977;170:10.

6. Teng CC, Chi HH, Katzin HM. Histology and mechanism of filtering operations. Am J Ophthalmol 1959;47:16-33.

7. Kee C. Prevention of early postoperative hypotony by partial ligation of silicone tube in Ahmed glaucoma valve implantation. J Glaucoma 2001;110:466-9.

8. Feldman RM, Tabet RR. Needle revision of filtering blebs. J Glaucoma 2008;17:594-600.

9. Emi K, Pederson JE, Toris CB. Hydrostatic pressure of the suprachoroidal space. Invest Ophthalmol Vis Sci 1989;30:233-238.

10. Melamed S, Ben Simon GJ, Goldenfeld M, et al. Efficacy and safety of gold micro shunt implantation to supraciliary space in patients with glaucoma: a pilot study. Arch Ophthalmol 2009;127:264-9.

11. Picht G, Grehn F. Development of the filtering bleb after trabeculectomy. Classification, histopathology, wound healing process. Ophthalmologe 1998;95:W380-W387.

12. Edmunds B, Bunce CV, Thompson JR, et al. Factors associated with success in first-time trabeculectomy for patients at low risk of failure with chronic open-angle glaucoma. Ophthalmology 2004;111:97-103. 
13. Mastropasqua L, Nubile M, Lanzini M, et al. Corneal and conjunctival manifestations in Fabry Disease: in vivo Confocal Microscopy study. Am J Ophthalmol 2006;141:709-718.

14. Labbé A, Dupas B, Hamard P, et al. In vivo confocal microscopy study of blebs after filtering surgery. Ophthalmology 2005; 112:1979.

15. Messmer EM, Zapp DM, Mackert MJ, et al. In vivo confocal microscopy of filtering blebs after trabeculectomy. Arch Ophthalmol 2006;124:1095-1103.

16. Guthoff R, Klink T, Schlunck G, et al. In Vivo Confocal Microscopy of Failing and Functioning Filtering Blebs: Results and Clinical Correlations. J Glaucoma 2006;15:552-558.

17. Ciancaglini M, Carpineto P, Agnifili L et al. Filtering Bleb Functionality: a Clinical, Anterior Segment Optical Coherence Tomography and In Vivo Confocal Microscopy Study. J Glaucoma 2009;17:308-17.

18. Wells A, Ashraff N, Hall R, et al. Comparison of Two Clinical Bleb Grading Systems. Ophthalmology 2006;113:77-83.

19. Azuara-Blanco A, Katz LJ. Disfunctional filtering blebs. Surv Ophthalmol 1998;43:93-126.

20. Epstein E: Fibrosis response to aqueous. Its relationship to glaucoma. Br J Ophthalmol $1959 ; 43: 641-7$.

21. Tsai JC, Johnson CC, Dietrich MS: The Ahmed shunt versus the Baerveldt shunt for refractory glaucoma - A single-surgeon comparison of outcome. Ophthalmology 2003;110:1814-21.

22. Amar N, LabbéA, Hamard P, et al. Filtering Blebs and Aqueous Pathway. An Immunocytological and In Vivo Confocal Microscopy. Ophthalmology 2008;115:1154-1161.

23. Ciancaglini M, Carpineto P, Agnifili L, et al. Conjunctival Modifications in Ocular Hypertension and Primary Open Angle Glaucoma: An In Vivo Confocal Microscopy Study. Invest Ophthalmol Vis Sci 2008;49:3042-3048.

24. Ciancaglini M, Carpineto P, Agnifili L et al. Conjunctival characteristics in primary open-angle glaucoma and modifications induced by trabeculectomy with mitomycin C: an in vivo confocal microscopy study. Br J Ophthalmol 2009;93:1204-9. 
Tables

Table 1: Patient demographic and clinical characteristics

\begin{tabular}{|c|c|c|c|}
\hline & $\begin{array}{c}\text { Successful } \\
\text { Implantations } \\
\text { (Group 1) } \\
\end{array}$ & $\begin{array}{c}\text { Failed } \\
\text { Implantations } \\
(\text { Group 2) } \\
\end{array}$ & p-value \\
\hline Number of eyes & $8 / 14$ & $6 / 14$ & \\
\hline Mean age (years) & $64.6 \pm 13.75$ & $64.6 \pm 9.95$ & ns* \\
\hline Gender (M/F) & $6 / 4$ & $2 / 2$ & ns** \\
\hline Pre-operative mean IOP $(\mathrm{mmHg})$ & $28.1 \pm 5.0$ & $29.6 \pm 1.9$ & ns* \\
\hline Post-operative mean IOP $(\mathrm{mmHg})$ & $14.3 \pm 2.8$ & $32.3 \pm 8.0$ & $\mathbf{P}=0.002 *$ \\
\hline Pre-operative medications & $2.7 \pm 0.9$ & $3.0 \pm 0.6$ & ns* \\
\hline Post-operative medications & $2.2 \pm 1.0$ & $2.8 \pm 0.4$ & ns* \\
\hline IVCM assessment after surgery (mo) & $15,5 \pm 5,52$ & $15,3 \pm 5,9$ & ns* \\
\hline Pre-operative drug regimen & & & ns* \\
\hline$\beta$-blockers & 8 & 6 & \\
\hline Prostaglandin derivatives & 6 & 6 & \\
\hline$\alpha_{2}$ agonists & 3 & 2 & \\
\hline $\mathbf{C A I}_{\mathrm{s}}$ & 5 & 4 & \\
\hline Duration of pre-operative therapy (mo) & $86.8 \pm 14.2$ & $89.1 \pm 13.3$ & $\mathbf{n s}^{*}$ \\
\hline
\end{tabular}

M=male; F=female; GMS=Gold Micro Shunt; $I O P=$ intraocular pressure; mo= months; $\mathbf{n s =}$ not significant

* T test for unpaired data

** Chi square test

$\mathrm{CAI}_{\mathrm{s}}=$ carbonic anhydrase inhibitors

IVCM: in vivo confocal microscopy 
Table 2: In Vivo Confocal Microscopy (IVCM) parameters

\begin{tabular}{lccc}
\hline & $\begin{array}{c}\text { Successful } \\
\text { Implantations } \\
\text { (Group 1) }\end{array}$ & $\begin{array}{c}\text { Failed } \\
\text { Implantations } \\
\text { (Group 2) }\end{array}$ & p-value \\
\hline $\begin{array}{l}\text { Mean microcysts density (MMD) } \\
\left(\text { cysts/mm } / \mathbf{m m}^{2}\right)\end{array}$ & $\mathbf{1 0 3 . 1 \pm 2 2 . 6}$ & $\mathbf{2 2 . 4 \pm 1 1 . 9}$ & $\mathbf{P}<0.01 *$ \\
$\begin{array}{l}\text { Mean microcysts area (MMA) } \\
\left(\text { cysts } / \mathbf{\mu m}^{2}\right)\end{array}$ & $\mathbf{2 9 4 8 9 . 3 \pm 1 2 9 5 4 . 9}$ & $\mathbf{4 6 9 6 . 0 \pm 3 6 0 8 . 1}$ & $\mathbf{P}<0.01 *$ \\
\hline
\end{tabular}

IVCM= in vivo confocal microscopy

*U Mann-Whitney test 


\section{Figure Legends}

Figure 1. Functioning implantation. A. Biomicroscopic aspect of a functioning Gold Micro Shunt implant correctly positioned in the anterior chamber (proximal end) and in the suprachoroidal space (distal end). No signs of conjunctival filtering bleb are detectable. B. Anterior segment optical coherence tomography showing the Gold Micro Shunt in the anterior chamber (arrowhead) and in the suprachoroidal space (arrow), with a spongiform appearance of the overlying sclera and with the hypoechoic space posteriorly to the device (asterisk). C. In vivo features of conjunctival epithelium at the corresponding area of the device insertion, characterized by intra-epithelial microcysts (arrows) partially filled and surrounded by what appears to be inflammatory cells (arrowhead). Bar represents $100 \mu \mathrm{m}$.

Figure 2. Non functioning implantation. A. Biomicroscopic aspect of a non functioning Gold Micro Shunt implant correctly positioned in the anterior chamber (proximal end) and in the suprachoroidal space (distal end). No signs of conjunctival filtering bleb are detectable. B. Anterior segment optical coherence tomography showing the Gold Micro Shunt in the anterior chamber (arrowhead) and in the suprachoroidal space (arrow), with a tight appearance of the sclera overlying the device. C. In vivo aspect of conjunctival epithelium at the corresponding area of the device insertion, showing a diffuse hyper-reflectivity of the layer with one intra-epithelial microcyst (arrow) containing what appears to be an inflammatory cell (arrowhead). Bar represents $100 \mu \mathrm{m}$. 
B

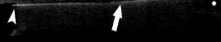




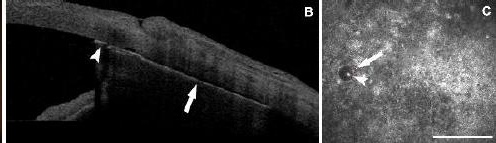

